\title{
Effects of salinity change on two superoxide dismutases (SODs) in juvenile marbled eel Anguilla marmorata
}

Li Wang, Xiaolu Wang, Shaowu Yin

Salinity is one of the most important factors that affect the fish growth and survival. Superoxide dismutases (SODs), as the primary antioxidant enzymes, play a first role in the process of preventing oxidative stress caused by excessive superoxide anion $\left(\mathrm{O}_{2}{ }^{-}\right)$in living organisms. In the present study, we investigated the effects of salinity on the gene expressions as well as enzymatic activities of MnSOD and Cu/ZnSOD in gill, intestine, kidney, liver and muscle tissues of marbled eel Anguilla marmorata. We found that liver might possess stronger redox capacity compared with other tissues. Furthermore, the gene expressions and enzymatic activities of SODs in juvenile marbled eels could be effectively enhanced by low salinity but inhibited when the salinity was higher than the body tolerance. Our findings indicated that MnSOD and $C U / Z n S O D$ played vital roles in the adaptation of marbled eels to salinity variation, which contributed to the elucidation of physiological adaptation and regulatory mechanism of SODs in eels. 
1 Effects of salinity change on two superoxide dismutases (SODs) in juvenile

2 marbled eel Anguilla marmorata

3 Short title: Salinity-adaptaion of SODs in marbled eel

$5 \quad$ Li Wang ${ }^{1,2, a}$, Xiaolu Wang ${ }^{1,2,}$, Shaowu Yin ${ }^{1,2}{ }^{*}$

$6 \quad{ }^{1}$ Jiangsu Key Laboratory for Biodiversity and Biotechnology, College of Life Sciences, Nanjing

7 Normal University, Nanjing, Jiangsu 210023, P. R. China

$8 \quad 2$ Co-Innovation Center for Marine Bio-Industry Technology of Jiangsu Province, Lianyungang,

9 Jiangsu 222005, P. R. China

$11{ }^{*}$ Corresponding author:

12 Shaowu Yin

13 College of Life Sciences, Nanjing Normal University, 1 Wenyuan Road, Nanjing, Jiangsu 21002,

14 P. R. China.

15 E-mail: yinshaowu@hotmail.com

aThese authors contributed equally to this work. 


\section{ABSTRACT}

24 Salinity is one of the most important factors that affect the fish growth and survival. Superoxide dismutases (SODs), as the primary antioxidant enzymes, play a first role in the process of preventing oxidative stress caused by excessive superoxide anion $\left(\mathrm{O}_{2}^{-}\right)$in living organisms. In the present study, we investigated the effects of salinity on the gene expressions as well as enzymatic activities of MnSOD and $\mathrm{Cu} / \mathrm{ZnSOD}$ in gill, intestine, kidney, liver and muscle tissues of marbled eel Anguilla marmorata. We found that liver might possess stronger redox capacity compared with other tissues. Furthermore, the gene expressions and enzymatic activities of SODs in juvenile marbled eels could be effectively enhanced by low salinity but inhibited when the salinity was higher than the body tolerance. Our findings indicated that $M n S O D$ and $\mathrm{Cu} / \mathrm{ZnSOD}$ played vital roles in the adaptation of marbled eels to salinity variation, which contributed to the elucidation of physiological adaptation and regulatory mechanism of SODs in eels.

36 Keywords: Salinity adaptation; Superoxide dismutases (SODs); Anguilla marmorata; Redox response 


\section{INTRODUCTION}

40 Salinity is one of the most important factors that affect fish growth and survival, since its variation may cause a series of physiological stress responses in aquatic animals, leading to imbalance of serum hormone, energy metabolism and electrolyte (Choi, An \& An, 2008). Recent studies have shown that stress responses caused by salinity variations are closely associated with enhanced generation of reactive oxygen species (ROS) (Livingstone, 2001). However, excessive ROS can lead to oxidative stress and cell malfunction, finally resulting in the apoptosis or necrosis (Hermes-Lima \& Zenteno-Savin, 2002; Sun et al., 2014). Organisms have developed defense mechanisms to shield themselves from such oxidative damage (Marikovsky et al., 2003).

Superoxide dismutase (SOD) is a key enzyme that can prevent oxidative stress through

1.15.1.1) can be classified into four distinct groups based on their structures, cellular 1987; Kim et al., 1998). intracellular environment (Chakravarthy et al., 2012), and MnSOD (Tian et al., 2011) plays a key antioxidant role in mitochondria (Cho et al., 2009). Previous studies have demonstrated that 
60 SOD expression is modulated by endotoxins (Cho et al., 2009; Sook Chung et al., 2012),

61 pathogens (Tian et al., 2011; Yu et al., 2011) and environmental pollution (Lopes et al., 2001),

62 suggesting a critical role of SODs in antioxidant system. In recent years, much attention has been

paid to the connection between salinity and antioxidant responses of fish (Ransberry et al., 2015;

Yin et al., 2011). The study on marine fish Pampus argenteus (Yin et al., 2011) showed that

certain low salinity can activate SOD, but its activity may be inhibited as the salinity drops

below its tolerance range. Moreover, in fish Pseudosciaena crocea (Wang et al., 2015), SOD

activity in the kidney is increased with reduction in salinity within a range from $7 \%$ o to $28 \%$.

However, most investigations in fish encountering salinity changes have focused on the changes

in the activities of antioxidant enzymes; nevertheless less attention has been paid to the

transcriptional level.

tropical catadromous fishes. This fish live widely across tropical and subtropical oceans and are

associated with fresh water (FW) systems. A. marmorata has been on the International Union for

Conservation of Nature Red List of Threatened Species due to over fishing and environmental

pollution, and it is regarded as species under the second-class national protection in China (Wang

et al., 2014). The life cycle of $A$. marmorata includes five stages as follows: leptocephalus, glass

eel, elver, yellow eel and silver eel, while A. marmorata must migrate from sea water (SW) to

FW for growth and development from the stage of elver (Li et al., 2015; Lin et al., 2012).

Although previous studies have shown that the antioxidant enzyme activity can be altered by

salinity changes (Yin et al., 2011; Wang et al., 2015), the regulatory mechanism of SODs in 
salinity adaptation of eels remains poorly understood.

In this study, we identified two $S O D$, denoted as $A m M n S O D$ and $A m C u / Z n S O D$. Moreover, we assessed their mRNA expression levels in eels in FW and analyzed the temporal mRNA expression profiles and enzymatic activity in vivo after they were transferred to brackish water (BW) and SW. Our results provided comparative perspectives into the two widespread and functional diverse enzymes, and offered important evidence to clarify the physiological adaptation and regulatory mechanism of SODs in eels.

\section{MATERIALS AND METHODS}

Juvenile A. marmorata $(18 \pm 0.81 \mathrm{~cm}$ in length, $18 \pm 0.77 \mathrm{~g}$ in weight) from $\mathrm{FW}$ were collected from Wenchang, Hainan Province, China by Hainan Wenchang Jinshan Eel Technology Co., Ltd. This company has obtained The People's Republic of China aquatic wild animal catching permit from Ministry of Agriculture of The People's Republic of China since 2004 (Approval number: National Fishery Resources and Environmental Protection 2004; 13). This study was also approved by the Ethics Committee of Experimental Animals at Nanjing Normal University (Research permit number: NNU20120301). All the eels were transferred to the tanks filled with filtered FW in the laboratory and fed to satiation with a commercial feed for eels every day. After acclimation at $25-26{ }^{\circ} \mathrm{C}$ for 1 week, they were used for the challenge experiments.

\section{SALINITY TREATMENT AND TISSUE SAMPLING}

The eels were divided into a control group and two experimental groups. The experimental groups consisted of BW (salinity of 10\%) and SW (salinity of 25\%) groups. In the control group, 
102

103

104

105

106

107

108

109

110

111

112

113

114

115

116

117

118

119

120

121

122

eels were reared in FW (salinity of $0 \%$ ). In BW and SW groups ( $\mathrm{n}=72$ for each group), the eels were primarily placed in FW, and then the salinity was gradually increased by $3 \%$ every day until it reached BW or SW. To evaluate the mRNA expression of two AmSODs under normal physiological condition, multiple tissues, including brain, gill, spleen, intestine, liver, kidney, muscle and heart, were collected from six eels in the control group. In order to determine the defense responses of $A m S O D s$ in these salinity adapted groups, multiple tissues, including gill, intestine, liver, kidney and muscle, were collected from six eels in the experimental groups at $1 \mathrm{~h}$, $3 \mathrm{~h}, 6 \mathrm{~h}, 12 \mathrm{~h}, 1$ and $2 \mathrm{~d}$ after the desired salinity was established. During the sampling process, experimental eels were anaesthetized with a solution of $0.05 \%$ MS-222 (Sigma, USA). In addition, the collected samples were also used to determine enzymatic activity of SODs. During the experimental period, salinity and $\mathrm{pH}(6.5-7.5)$ were monitored daily.

\section{TOTAL RNA EXTRACTION AND CDNA SYNTHESIS}

Total RNA was extracted from above-mentioned tissues using High Purity RNA Fast Extract Reagent (BioTeke, Beijing, China; http:/www.teke.bioon.com.cn/) according to the manufacturer's instructions, and extracted RNA was stored at $-80{ }^{\circ} \mathrm{C}$ before further analysis. The RNA concentration was determined using NanoDrop 2000 (Thermo, Wilmington, DE, USA), and its integrity was examined on $1.0 \%$ agarose gel. The single-strand cDNA was synthesized using HiScript ${ }^{\mathrm{TM}}$ QRT SuperMix (Vazyme, New Jersey, USA; http://www.vazyme.com/) for subsequent quantitative real-time PCR (qRT-PCR).

\section{ANALYSIS OF AMSOD EXPRESSION}

In our previous study, the full-length $A m M n S O D s$ and $A m C u / Z n S O D$ have been cloned using 
123

124

125

126

127

128

129

130

131

132

133

134

135

136

137

138

139

140

141

142

143

the $3^{\prime}$ and 5' rapid amplification cDNA end (RACE) method, and their NCBI accession no. was

KR350467 and KR350468, respectively (Wang et al., 2016). Tissue distribution and temporal expression profiles of $A m M n S O D$ and $A m C u / Z n S O D$ in eels under normal conditions (FW group) and eels with salinity treatment (BW and SW groups) were investigated by qRT-PCR. Table 1 lists all the gene-specific primers for $A m M n S O D, A m C u / Z n S O D$ and Am $\beta$-actin used in this study. The experiments were performed in a $20-\mu \mathrm{L}$ reaction system consisting of $4 \mu \mathrm{L}$ of diluted cDNA template, $10 \mu \mathrm{L}$ of Faststart Universal SYBR Green Master (Roche, Basel, Switzerland; http://www.roche.com/), $1 \mu \mathrm{L}$ of each primer $(6 \mathrm{mmol} / \mu \mathrm{L})$ and $4 \mu \mathrm{L} d \mathrm{ddH}_{2} \mathrm{O}$, and each experiment was performed in triplicate. Briefly, after a denaturation step at $94{ }^{\circ} \mathrm{C}$ for $10 \mathrm{~min}$, the amplification was carried out with 40 cycles at a melting temperature of $94{ }^{\circ} \mathrm{C}$ for $10 \mathrm{~s}$, an annealing temperature of $55{ }^{\circ} \mathrm{C}$ for $30 \mathrm{~s}$, and an extension temperature of $72{ }^{\circ} \mathrm{C}$ for $60 \mathrm{~s}$. A melting curve was generated after each reaction to confirm the efficiency of qRT-PCR, and absence of primer dimers or other non-specific products was also verified based on the analysis of the melting curve. The relative expression level of $A m S O D$ transcripts was determined by the $2^{-\Delta \Delta \mathrm{Ct}}$ comparative $\mathrm{Ct}$ method using $\beta$-actin as an internal control (Livak \& Schmittgen, 2001). The calculated relative expression level of $A m S O D s$ in each tissue was compared with its respective level in spleen in the tissue-specific expression analysis. In the time-course analysis, the fold-change post salinity treatment was determined by comparing with the expression level in FW group.

\section{MEASUREMENT OF SOD ENZYMATIC ACTIVITIES}

The SOD enzymatic activity in the above-mentioned tissues was determined using the SOD 
144 Typing Testing Kit (Jiancheng Bioengineering, Nanjing, China; http://www.njjcbio.com/) after

145 the salinity reached the target salinity. The experimental tissues were homogenized in normal

146 saline $[0.85 \%(\mathrm{w} / \mathrm{v})$ of $\mathrm{NaCl}$, denoted as $\mathrm{NS}]$ by an electric homogenizer. Coomassie Brilliant

147 Blue was used to determine the protein concentration in the crude extract according to the

148 manufacturer's protocol (Jiancheng Bioengineering, Nanjing, China). The total SOD enzymatic

149 activity and $\mathrm{Cu} / \mathrm{ZnSOD}$ activity were determined following the manufacturer's instructions. Each

150 sample was measured in triplicate.

151

152

153

\section{STATISTICAL ANALYSIS}

All data were expressed as mean $\pm \mathrm{SD}$. of triplicates, and the results were subjected to oneway analysis of variance (one-way ANOVA) and two-tailed paired t test with SPSS v17.0 software. A difference was considered to be statistically significant at $P<0.05$ and extremely significant at $P<0.01$.

\section{RESULTS}

\section{SPATIAL EXPRESSION AND TISSUE DISTRIBUTION OF AMSODS}

Fig. 1 shows that the mRNA expression levels of both SODs were detectable in all the eight tissues examined by qRT-PCR assay, but their relative baseline expression levels varied. Predominant expression of $A m M n S O D$ was detected in liver, muscle and heart tissues $(P<0.001$; 11.6-fold, 26.3-fold and 10.3-fold, respectively). In contrast, $A m C u / Z n S O D$ was highly expressed in liver $(P<0.001 ; 48.4$-fold). Moreover, the mRNA abundance of AmSODs was low in other tissues.

\section{TEMPORAL TRANSCRIPTIONAL REGULATION OF THE TWO AMSODS}


Although no mortality or pathologies during the experiment, our results clearly revealed that

the salinity variation significantly altered the expressions of AmSODs. In gill, kidney, liver and

muscle tissues [Fig. 2, 3(a, c, d and e)], the expression levels of $A m M n S O D$ and $A m C u / Z n S O D$

showed a trend of rising at first and then reducing with prolonged time in BW. However, the

expression levels of $A m M n S O D$ and $A m C u / Z n S O D$ were first decreased and then exhibited

an upward trend in gill, liver and muscle tissues in SW. However, the expression level of

$\mathrm{AmCu} / \mathrm{ZnSOD}$ was first increased and decreased afterwards in kidney in SW. In contrast, the

expression level of $A m M n S O D$ was significantly greater than that of the control group at $12 \mathrm{~h}$

and $2 \mathrm{~d}(\mathrm{P}<0.001)$ merely. In intestine [Fig. 2(b) and 3(b)], the mRNA levels of AmMnSOD and

$A m C u / Z n S O D$ were barely changed within $6 \mathrm{~h}$ in both BW and SW, and then both reached their

peak levels at $12 \mathrm{~h}$ in SW $(P<0.001)$. However, the expressions of AmMnSOD and

$A m C u / Z n S O D$ peaked at $1 \mathrm{~d}$ and $12 \mathrm{~h}(P<0.01)$ in BW, respectively. In addition, the expression

levels of $A m M n S O D$ in intestine and $A m C u / Z n S O D$ in kidney were significant higher in SW

compared with BW. In comparison, the expression levels of AmMnSOD in gill and

$A m C u / Z n S O D$ in muscle were significant higher at $6 \mathrm{~h}$ and $2 \mathrm{~d}$ in $\mathrm{SW}$ compared with BW,

respectively. However, their expression levels were significant lower at $1 \mathrm{~d}$ in SW compared

with BW. Moreover, the expression levels of $A m M n S O D$ (kidney, liver and muscle) and

$\mathrm{AmCu} / \mathrm{ZnSOD}$ (gill, intestine and liver) were significant higher in BW compared with SW.

\section{CHANGES IN SOD ENZYMATIC ACTIVITIES}

184 In order to examine the antioxidant status in A. marmorata in response to different salinity 185

levels, we determined the AmSOD enzymatic activities in different treatment groups. 
Fig. 4 and 5 show that the variation trend of total SOD activity was similar to that of

$\mathrm{Cu} / \mathrm{ZnSOD}$ activity in intestine, kidney and muscle tissues. However, a significantly different variation trend was observed between the total SOD activity and $\mathrm{Cu} / \mathrm{ZnSOD}$ activity at $2 \mathrm{~d}$ in gill and liver tissues of BW group [Fig. 4, 5(a) and (d)]. Moreover, the $\mathrm{Cu} / \mathrm{ZnSOD}$ activity in gill was significantly decreased at first and then increased in $\mathrm{BW}$, while the $\mathrm{Cu} / \mathrm{ZnSOD}$ activity in intestine, kidney, liver and muscle tissues was first increased and then decreased. Fig. 5(a), (c) and (e) show that the changes of $\mathrm{Cu} / \mathrm{ZnSOD}$ activities were similar in gill, kidney and muscle tissues in SW, exhibiting an overall decreasing trend within 2 d. Fig. 5(b) shows that the $\mathrm{Cu} / \mathrm{ZnSOD}$ activity in intestine was maintained at the basal level $(P<0.05)$. Conversely, the $\mathrm{Cu} / \mathrm{ZnSOD}$ activity was distinctively altered in liver, showing a significant up-regulation from 1

h to $1 \mathrm{~d}(P<0.05)$ in SW [Fig. 5(d)]. In addition, the total SOD and Cu/ZnSOD activities in kidney and muscle tissues exhibited an overall higher level within $2 \mathrm{~d}$ in BW compared with SW.

In contrast, the total $\mathrm{SOD}$ and $\mathrm{Cu} / \mathrm{ZnSOD}$ activities in other tissues in $\mathrm{BW}$ were only higher at several time points compared with SW, such as in gill and intestine tissues. Moreover, the total SOD activity in liver showed a lower level within $2 \mathrm{~d}$ in BW compared with SW.

\section{DISCUSSION}

All the experimental fish were denoted as juvenile eel by measuring their body weight and length. Therefore, they should be more easily threatened by salinity pressure compared with adult $A$. marmorata. What' more, our preliminary experiment also revealed the rapid salinity increase of water environment may lead to death of experimental fish, and the similar results 
207 Previous study has indicated that $A$. marmorata must adapt three types of water environment 208 during migration process, namely Freshwater, Brackish water and Seawater (Lin et al., 2012).

209 Therefore, we chose three special salinity of $0 \%, 10 \%$ and $25 \%$ as the representation of the FW, 210 BW and SW respectively, and we used the oxidative and antioxidant relative tissues, such as 211 liver, kidney, gill, intestine and muscle, to investigate the redox capacity of MnSOD and $212 \mathrm{Cu} / \mathrm{ZnSOD}$ of marbled eels in three different salinity range of water environment.

213 Liver, kidney and intestine have high metabolic rate, which is important to maintain steadystate and normal physiological function for fish, and liver is a vital organ for detoxification and xenobiotic metabolism (Lushchak, 2015; Sun et al., 2014). Some previous studies suggest that multiple oxidative reactions and antioxidant defenses also occur in gill and muscle tissues

(Ahmad et al., 2006; Yin et al., 2011). A similar tissue distribution profile of AmMnSOD has been reported for the MnSOD in fish Megalobrama amblycephala (Yih 1955) (Sun et al., 2014) and mollusc Mytilus galloprovincialis (Lamarck 1819) (Wang et al., 2013), in which a higher expression level is observed in liver and muscle. In addition, the spatial expression pattern of $A m C u / Z n S O D$ was similar to that of $C u / Z n S O D$ in fish Pseudosciaena crocea (Richardson 1846)

(Liu et al., 2015) and fish Hypophthalmichthys molitrix (Valenciennes 1844) (Zhang et al., 2011).

Therefore, the differential expressions of $A m S O D s$ in gill, intestine, kidney, liver and muscle tissues clearly suggested that the expression level of AmSODs was closely related to the antioxidant responses of marbled eels in comparative perspective.

To the best of our knowledge, this is the first report systemically investigating the mRNA expression changes of the two SODs in different tissues following the environment transfer from 
$228 \mathrm{FW}$ to $\mathrm{BW}$ and then to SW. Our results clearly revealed that the salinity variation significantly

229 altered the expressions of AmSODs. Fig. 2 and 3 show that both $A m S O D s$ were significantly up-

230 regulated in gill, kidney, liver and muscle tissues at early-phases in BW, indicating that these

231 tissues were more sensitive to salt-induced alterations in low level than intestine. Previous study

232 has also shown that liver, kidney and muscle tissues are considered highly susceptible to salinity

233 changes (Zhao et al., 2008). However, the expression levels of AmSODs in gill, liver and muscle

234 tissues were inhibited at early-phase in SW, and the $A m S O D$ expression reached its peak at late-

235 phase after acclimatization of $12 \mathrm{~h}$ to $1 \mathrm{~d}$. These results suggested that the antioxidant response

236 susceptibility of marbled eels was different when exposed to different salinity stresses.

237 Furthermore, low salinity might stimulate the mRNA expression levels of $M n S O D$ and

$238 \mathrm{Cu} / \mathrm{ZnSOD}$, while the high salinity might inhibit their expressions.

239 Consistent with the changes of $A m S O D s$ at the mRNA level in BW, their expressions at the

240 enzymatic activity level appeared to be significantly elevated with the prolonged induction time

241 at the early phase. However, $\mathrm{Cu} / \mathrm{ZnSOD}$ in liver peaked at $6 \mathrm{~h}$ in $\mathrm{BW}$ at the enzymatic activity

242 level, while its mRNA level peaked at $1 \mathrm{~h}$ after salinity exposure, suggesting that the relation

243 between enzymatic activity and mRNA expression was not strictly linear, and enzymatic activity

244 is controlled by gene expression as well as enzyme activation (Chambers \& Matrisian, 1997).

245 The observed lag between the two molecules was probably due to the time difference for de novo

246 synthesis of SOD proteins. Previous study has also shown that SOD enzymatic activity is lagged

247 compared with its expression at the mRNA level (Wang et al., 2016). Interestingly, the

$248 \mathrm{Cu} / \mathrm{ZnSOD}$ activity in gill, kidney and muscle tissues was significantly up-regulated in BW, 
249 while it was down-regulated in SW compared with FW. These results could be explained by that

250 low salinity stimulates SODs to defend against excessive ROS-induced damage, but their

251 activities may be inhibited once the salinity is above their tolerance range (Yin et al., 2011),

252 which partly explains the fatality occurring in juvenile fish Pampus argenteus (Yin et al., 2010).

253 In addition, in intestine of treated eels, the expression levels of two AmSODs peaked at $12 \mathrm{~h}$ in

254 BW and SW, while the total SOD activity was significantly up-regulated at early-phase in BW

255 and SW, indicating the strong antioxidant responses in intestine of eels when exposed to 256 different salinities.

257 In conclusion, based on the expression profiles of AmSODs at the mRNA and enzymatic activity levels after salinity exposure, we supposed that SODs in juvenile marbled eels could be effectively enhanced by low salinity but inhibited when the salinity was higher than the body tolerance. Besides, in the total SOD enzymatic activity and $\mathrm{Cu} / \mathrm{ZnSOD}$ activity levels, only the SOD activities in liver could keep an up-regulated trend within $2 \mathrm{~d}$ in SW, while those in gill, kidney, intestine and muscle tissues were inhibited in varying degrees. Therefore, we inferred that liver might possess stronger redox capacity compared with other tissues.

264 In the present study, we identified two SODs, denoted as $A m M n S O D$ and $A m C u / Z n S O D$.

Moreover, we assessed their mRNA expression levels in eels in FW and analyzed the temporal mRNA expression profiles and enzymatic activity in vivo after they were transferred to BW and SW. All these results indicated that $A m M n S O D$ and $A m C u / Z n S O D$ played vital roles in the adaptation of marbled eels to salinity variation. Moreover, our findings provided new and valuable evidence to further clarify the physiological adaptation and regulatory mechanism of 
270 SODs in eels.

271 ACKNOWLEDGEMENTS

272 The authors thank Xiaolu Wang and Shaowu Yin for field and laboratory support.

273 ADDITIONAL INFORMATION AND DECLARATIONS

274

DNA Deposition

275 The following information was supplied regarding the deposition of DNA sequences:

276 GenBank. Accession numbers: KR350467- KR350468.

277

278

279

280

281

282

283

284

285

286

287

288

289

290

\section{REFERENCES}

Ahmad I, Maria VL, Oliveira M, Pacheco M, and Santos MA. 2006. Oxidative stress and genotoxic effects in gill and kidney of Anguilla anguilla L. exposed to chromium with or without pre-exposure to beta-naphthoflavone. Mutation Research-Genetic Toxicology and Environmental Mutagenesis 608:16-28 DOI 10.1016/j.mrgentox.2006.04.020.

Bannister JV, Bannister WH, and Rotilio G. 1987. Aspects of the structure, function, and applications of superoxide dismutase. CRC Critical Reviews Biochemistry 22:111-180 DOI 10.3109/10409238709083738.

Chakravarthy N, Aravindan K, Kalaimani N, Alavandi SV, Poornima M, and Santiago TC. 2012. Intracellular Copper Zinc Superoxide dismutase (icCuZnSOD) from Asian seabass (Lates calcarifer): molecular cloning, characterization and gene expression with reference to Vibrio anguillarum infection. Developmental and Comparative Immunology 36:751-755 DOI 10.1016/j.dci.2011.11.002.

Chambers AF, and Matrisian LM. 1997. Changing views of the role of matrix metalloproteinases 
in metastasis. Journal of the National Cancer Institute 89:1260-1270 DOI 10.1093/jnci/89.17.1260.

293

294

295

296

297

298

299

300

301

302

303

304

305

306

307

308

309

310

311

Cho YS, Lee SY, Bang IC, Kim DS, and Nam YK. 2009. Genomic organization and mRNA expression of manganese superoxide dismutase (Mn-SOD) from Hemibarbus mylodon (Teleostei, Cypriniformes). Fish and Shellfish Immunology 27:571-576 DOI 10.1016/j.fsi.2009.07.003.

Choi CY, An KW, and An MI. 2008. Molecular characterization and mRNA expression of glutathione peroxidase and glutathione S-transferase during osmotic stress in olive flounder (Paralichthys olivaceus). Comparative Biochemistry and Physiology Part A: Molecular and Integrative Physiology 149:330-337 DOI 10.1016/j.cbpa.2008.01.013.

Hermes-Lima M, and Zenteno-Savin T. 2002. Animal response to drastic changes in oxygen availability and physiological oxidative stress. Comparative Biochemistry and Physiology Part C: Toxicology \& Pharmacology 133:537-556 DOI: 10.1016/S1532-0456(02)000807.

Kim EJ, Chung HJ, Suh B, Hah YC, and Roe JH. 1998. Transcriptional and post-transcriptional regulation by nickel of sodN gene encoding nickel-containing superoxide dismutase from Streptomyces coelicolor Muller. Molecular Microbiology 27:187-195 DOI 10.1046/j.1365-2958.1998.00674.x.

Li L, Jia YH, Li P, Yin SW, Zhang GS, Wang XL, Wang YY, and Wang XJ. 2015. Molecular cloning, characterization, and expression of vacuolar-type-H+-ATPase B1 (VHAB1) gene in the gill of Anguilla marmorata. Genetics and Molecular Research 14:8008-8020 
313 Li Z, Lui EY, Wilson JM, Ip YK, Lin QS, Lam TJ, and Lam SH. 2014. Expression of key ion transporters in the gill and esophageal-gastrointestinal tract of euryhaline mozambique tilapia oreochromis mossambicus acclimated to fresh water, seawater and hypersaline water. Plos One 9:e87591-e87591 DOI 10.1371/journal.pone.0087591.

Liu H, He J, Chi C, and Gu Y. 2015. Identification and analysis of icCu/Zn-SOD, Mn-SOD and ecCu/Zn-SOD in superoxide dismutase multigene family of Pseudosciaena crocea. Fish and Shellfish Immunology 43:491-501 DOI 10.1016/j.fsi.2015.01.032.

Livak KJ, and Schmittgen TD. 2001. Analysis of relative gene expression data using real-time quantitative PCR and the 2(T)(-Delta Delta C) method. Methods 25:402-408 DOI 10.1006/ meth.2001.1262, available online at http:/ / www.idealibrary.com on.

Livingstone DR. 2001. Contaminant-stimulated reactive oxygen species production and oxidative damage in aquatic organisms. Marine Pollution Bulletin 42:656-666 DOI 10.1016/S0025-326X(01)00060-1.

Lopes PA, Pinheiro T, Santos MC, da Luz Mathias M, Collares-Pereira MJ, and Viegas-Crespo AM. 2001. Response of antioxidant enzymes in freshwater fish populations (Leuciscus 
333

334

335

336

337

338

339

340

341

342

343

344

345

346

347

348

349

350

351

352

353

alburnoides complex) to inorganic pollutants exposure. Science of the Total Environment 280:153-163 DOI 10.1016/S0048-9697(01)00822-1.

Lushchak VI. 2015. Contaminant-induced oxidative stress in fish: a mechanistic approach. Fish Physiology and Biochemistry DOI 10.1007/s10695-015-0171-5.

Marikovsky M, Ziv V, Nevo N, Harris-Cerruti C, and Mahler O. 2003. Cu/Zn superoxide dismutase plays important role in immune response. Journal of Immunology 170:29933001 DOI: 10.4049/jimmunol.170.6.2993.

Ransberry VE, Morash AJ, Blewett TA, Wood CM, and McClelland GB. 2015. Oxidative stress and metabolic responses to copper in freshwater- and seawater-acclimated killifish, Fundulus heteroclitus. Aquatic Toxicology 161:242-252 DOI 10.1016/j.aquatox.2015.02.013.

Sook Chung J, Bachvaroff TR, Trant J, and Place A. 2012. A second copper zinc superoxide dismutase (CuZnSOD) in the blue crab Callinectes sapidus: cloning and up-regulated expression in the hemocytes after immune challenge. Fish and Shellfish Immunology 32:16-25 DOI 10.1016/j.fsi.2011.08.023.

Sun S, Zhu J, Jiang X, Li B, and Ge X. 2014. Molecular cloning, tissue distribution and expression analysis of a manganese superoxide dismutase in blunt snout bream Megalobrama amblycephala. Fish and Shellfish Immunology 38:340-347 DOI 10.1016/j.fsi.2014.03.036.

Tian J, Chen J, Jiang D, Liao S, and Wang A. 2011. Transcriptional regulation of extracellular copper zinc superoxide dismutase from white shrimp Litopenaeus vannamei following 
354

355

356

357

358

359

360

361

362

363

364

365

366

367

368

369

370

371

372

373

374

Vibrio alginolyticus and WSSV infection. Fish and Shellfish Immunology 30:234-240

DOI 10.1016/j.fsi.2010.10.013.

Vaughan M. 1997. Oxidative Modification of Macromolecules Minireview Series. Journal of Biological Chemistry 272:18513-18513 DOI 10.1074/jbc.272.30.18513.

Wang Q, Yuan Z, Wu H, Liu F, and Zhao J. 2013. Molecular characterization of a manganese superoxide dismutase and copper/zinc superoxide dismutase from the mussel Mytilus galloprovincialis. Fish and Shellfish Immunology 34:1345-1351 DOI 10.1016/j.fsi.2013.01.011.

Wang XL, Wang L, Ren Q, Yin SW, Liang FF, and Jia YH. 2016. Two superoxide dismutases (SODs) respond to bacterial challenge identified in the marbled eel Anguilla marmorata. Aquaculture 451:316-325 DOI 10.1016/j.aquaculture.2015.09.025.

Wang XL, Xu F, Li L, Zhang YP, Ding YD, Zang X, Jia YH, Zhang GS, Wang YY, Wang XJ, Zhang HW, Yin SW. 2014. Development of 39 novel polymorphic microsatellite markers for the giant mottled eel Anguilla marmorata and cross-amplification in other eel species. Conservation Genetics Resources 6:865-871 DOI 10.1007/s12686-014-0227-3.

Wang Y, Li W, Li L, Zhang W, and Lu W. 2015. Effects of salinity on the physiological responses of the large yellow croaker Pseudosciaena crocea under indoor culture conditions. Aquaculture Research:n/a-n/a DOI 10.1111/are.12788.

Yin F, Peng S, Sun P, and Shi Z. 2011. Effects of low salinity on antioxidant enzymes activities in kidney and muscle of juvenile silver pomfret Pampus argenteus. Acta Ecologica Sinica 31:55-60 DOI 10.1016/j.chnaes.2010.11.009. 
375 Yin F, Peng SM, Sun P, and Shi ZH. 2010. Effect of low salinity on digestive enzyme activities

376

377

378

379

380

381

382

383

384

385

386

387

388

389

390

391

392

393

394 395 in the intestinal tract of juvenile silver pomfret Pampus argenteus. Marine Fisheries. 32:160-165 DOI 10.13233/j.cnki.mar.fish.2012.02.008.

Yu Z, He X, Fu D, and Zhang Y. 2011. Two superoxide dismutase (SOD) with different subcellular localizations involved in innate immunity in Crassostrea hongkongensis. Fish and Shellfish Immunology 31:533-539 DOI 10.1016/j.fsi.2011.06.022.

Zhang ZW, Li Z, Liang HW, Li L, Luo XZ, and Zou GW. 2011. Molecular cloning and differential expression patterns of copper/zinc superoxide dismutase and manganese superoxide dismutase in Hypophthalmichthys molitrix. Fish and Shellfish Immunology 30:473-479 DOI 10.1016/j.fsi.2010.11.003.

Zhao F, Zhuang P, Zhang LZ, Huang XR, Zhang T, and Feng GP. 2008. Responses of antioxidases in different tissues of Acipenser schrenckii to increased salinity in water. Marine Fisheries Research 29:65-69 DOI 10.3969/j.issn.1000-7075.2008.05.010.

Table 1. List of primers used in this study.

\section{Figure legends}

Fig. 1 Tissue distribution analysis of $A m M n S O D$ and $A m C u / Z n S O D$ at the mRNA level of $A$. marmorata

The relative mRNA expression levels of $A m S O D s$ in each tissue were calculated by the $2^{-\Delta \Delta \mathrm{Ct}}$ method using $A$. marmorata $\beta$-actin as an internal reference gene. Vertical bars represent the S.D. 
$396(n=3)$. Data indicated with asterisk symbol $\left(^{*}\right)$ are significantly different from the spleen tissue, *

397 at $P<0.05, * *$ at $P<0.01$ and $* * *$ at $P<0.001$.

398

399

Fig. 2 Temporal mRNA expression analysis of $A m M n S O D$ in gill (A), intestine (B), kidney (C),

liver (D) and muscle tissues (E) of juvenile eels due to the change of salinity

401

The relative mRNA expression level of $A m M n S O D$ in each tissue was determined using $A$.

marmorata $\beta$-actin as an internal reference gene. Then the expression levels of $A m M n S O D$ were

403

presented as fold-change relative to FW. Vertical bars represent the S.D. $(n=3)$. Data indicated

with asterisk symbol (*) are significantly different from the corresponding FW control and data

with asterisk symbol $(*)$ on the box indicated significant difference between $\mathrm{BW}$ and $\mathrm{SW},{ }^{*}$ at $P$

406

$<0.05, * *$ at $P<0.01$ and $* * *$ at $P<0.001$.

Fig. 3 Temporal mRNA expression analysis of $A m C u / Z n S O D$ in gill (A), intestine (B), kidney

(C), liver (D) and muscle tissues (E) of juvenile eels due to the change of salinity

410

The relative mRNA expression level of $A m C u / Z n S O D$ in each tissue was determined using $A$.

marmorata $\beta$-actin as an internal reference gene. Then the expression levels of $A m C u / Z n S O D$

were presented as fold-change relative to FW. Vertical bars represent the S.D. $(n=3)$. Data

indicated with asterisk symbol (*) are significantly different from the corresponding FW control

and data with asterisk symbol (*) on the box indicated significant difference between BW and

$\mathrm{SW}, *$ at $P<0.05, * *$ at $P<0.01$ and $* * *$ at $P<0.001$. 
417 Fig. 4 The total SOD enzymatic activity (U/mg protein) analysis of $A$. marmorata in gill (A),

418 intestine (B), kidney (C), liver (D) and muscle tissues (E) of juvenile eels in response to BW and

419 SW adaptation. Vertical bars represent the S.D. $(n=3)$. Data indicated with asterisk symbol $(*)$

420 are significantly different from corresponding FW group and data with asterisk symbol (*) on the

421 box indicated significant difference between $\mathrm{BW}$ and $\mathrm{SW}, *$ at $P<0.05$.

Fig. 5 The CuZnSOD enzymatic activity (U/mg protein) analysis of $A$. marmorata in gill (A),

424 intestine (B), kidney (C), liver (D) and muscle tissues (E) of juvenile eels in response to BW and

425 SW adaptation. Vertical bars represent the S.D. $(n=3)$. Data indicated with asterisk symbol $(*)$

426 are significantly different from corresponding FW group and data with asterisk symbol $(*)$ on the

427 box indicated significant difference between $\mathrm{BW}$ and $\mathrm{SW}, *$ at $P<0.05$. 


\section{Table 1 (on next page)}

List of primers used in this study 
1 Table 1. List of primers used in this study.

2

3

4

\begin{tabular}{lll}
\hline Primer Name & Purpose & Primer sequence (5'-3') \\
\hline CuZnSOD-F & qRT-PCR amplification & CTTCAACCCGCACAACAAGA \\
CuZnSOD-R & qRT-PCR amplification & TGCCGGTTTTCAAGCTTTCA \\
MnSOD-F & qRT-PCR amplification & CACAGCAAACACCACGCC \\
MnSOD-R & qRT-PCR amplification & TGGACATCTTCTCCCTCAGC \\
Am $\beta$-actin-F & qRT-PCR internal reference & GCAGATGTGGATCAGCAAGC \\
Am $\beta$-actin-R & qRT-PCR internal reference & ACATTGCCGTCACCTTCATGC \\
\hline
\end{tabular}


1

Tissue distribution analysis of AmMnSOD and AmCu/ZnSOD at the mRNA level of A. marmorata[i]

The relative mRNA expression levels of AmSODs in each tissue were calculated by the $2^{-\Delta \Delta C t}$ method using A. marmorata $\beta$-actin as an internal reference gene. Vertical bars represent the S.D. $(n=3)$. Data indicated with asterisk symbol $(*)$ are significantly different from the spleen tissue, $*$ at $P<0.05$, ** at $P<0.01$ and ${ }^{* * *}$ at $P<0.001$.

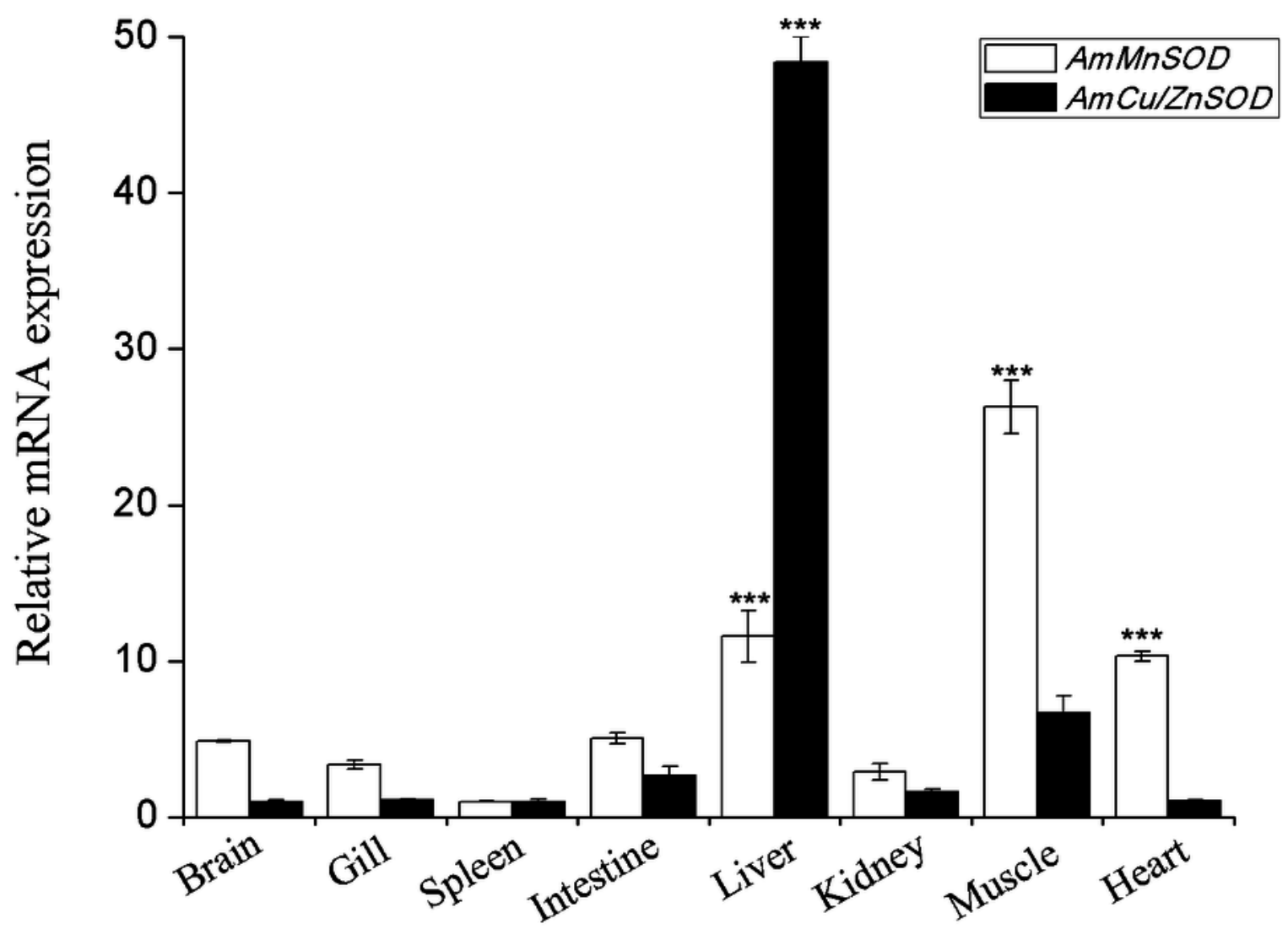


2

Temporal mRNA expression analysis of AmMnSOD[i] in gill (A), intestine (B), kidney (C), liver (D) and muscle tissues (E) of juvenile eels due to the change of salinity

The relative mRNA expression level of AmMnSOD in each tissue was determined using $A$. marmorata $\beta$-actin as an internal reference gene. Then the expression levels of AmMnSOD was presented as fold-change relative to FW. Vertical bars represent the S.D. $(n=3)$. Data indicated with asterisk symbol (*) are significantly different from the corresponding FW control and data with asterisk symbol $(*)$ on the box indicated significant difference between BW and SW, * at $\mathrm{P}<0.05$, ** at $P<0.01$ and $* * *$ at $\mathrm{P}[\mathrm{i}]<0.001$. [b]
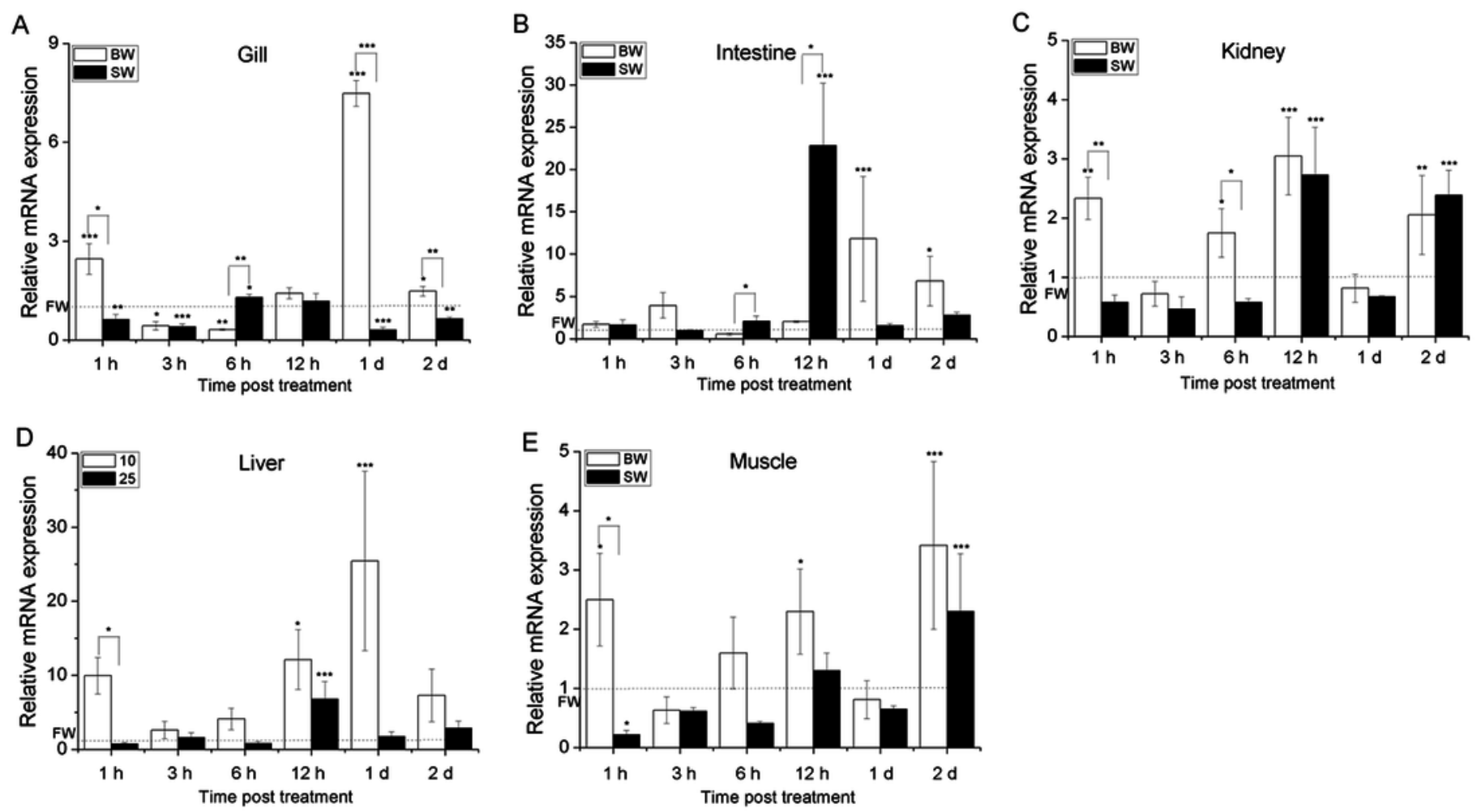


\section{3}

Temporal mRNA expression analysis of AmCu/ZnSOD[i] in gill (A), intestine (B), kidney $(C)$, liver (D) and muscle tissues (E) of juvenile eels due to the change of salinity

The relative mRNA expression level of AmCu/ZnSOD in each tissue was determined using $A$. marmorata $\beta$-actin as an internal reference gene. Then the expression levels of $\mathrm{AmCu} / \mathrm{ZnSOD}$ was presented as fold-change relative to FW. Vertical bars represent the S.D. $(n=3)$. Data indicated with asterisk symbol $(*)$ are significantly different from the corresponding FW control and data with asterisk symbol $(*)$ on the box indicated significant difference between $\mathrm{BW}$ and $\mathrm{SW}, *$ at $\mathrm{P}<0.05$, ** at $\mathrm{P}<0.01$ and $* * *$ at $\mathrm{P}[\mathrm{i}]<0.001$. [b]
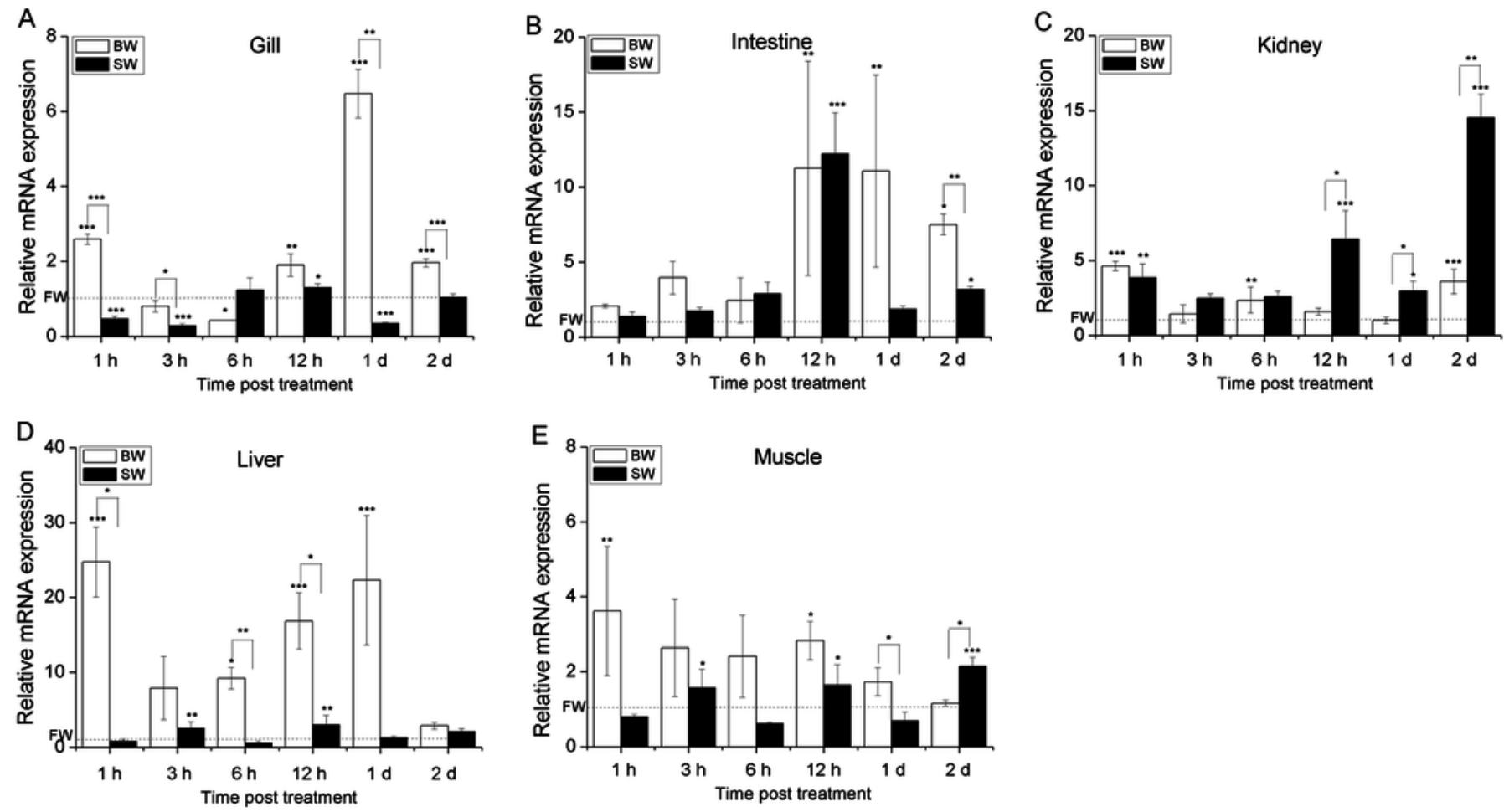
4

The total SOD enzymatic activity analysis of $A$. marmorata

The total SOD enzymatic activity ( $U / m g$ protein) analysis of $A$. marmorata in gill (A), intestine (B), kidney (C), liver (D) and muscle tissues (E) of juvenile eels in response to BW and SW adaptation. Vertical bars represent the S.D. $(n=3)$. Data indicated with asterisk symbol (*) are significantly different from corresponding FW group and data with asterisk symbol $(*)$ on the box indicated significantdifference between BW and SW, * at $P<0.05$.
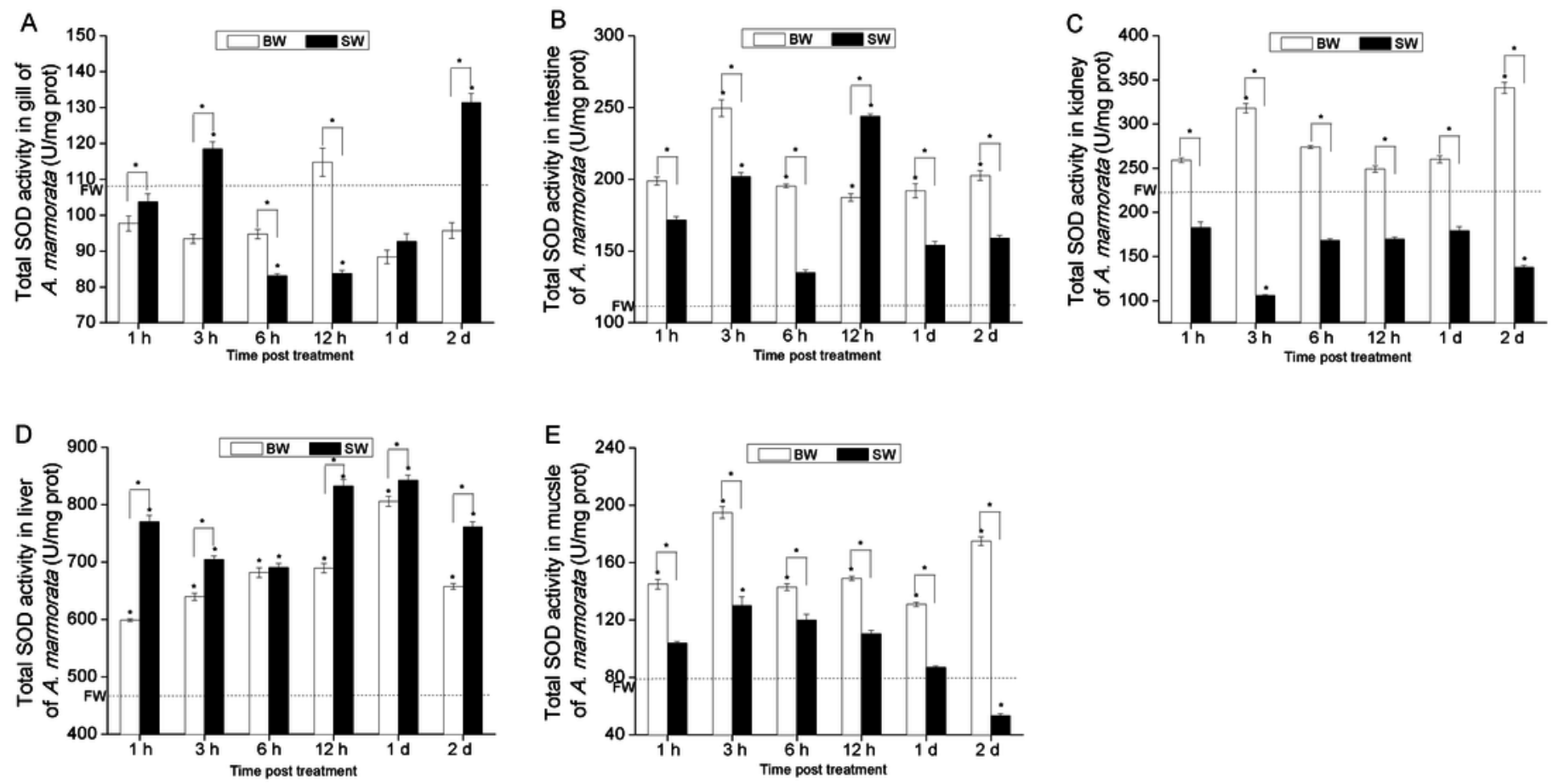


\section{5}

The CuZnSOD enzymatic activity analysis of $A$. marmorata

The CuZnSOD enzymatic activity ( $U / m g$ protein) analysis of $A$. marmorata in gill (A), intestine (B), kidney (C), liver (D) and muscle tissues (E) of juvenile eels in response to BW and SW adaptation. Vertical bars represent the S.D. $(n=3)$. Data indicated with asterisk symbol $(*)$ are significantly different from corresponding FW group and data with asterisk symbol $(*)$ on the box indicated significantdifference between BW and SW, * at $P<0.05$.
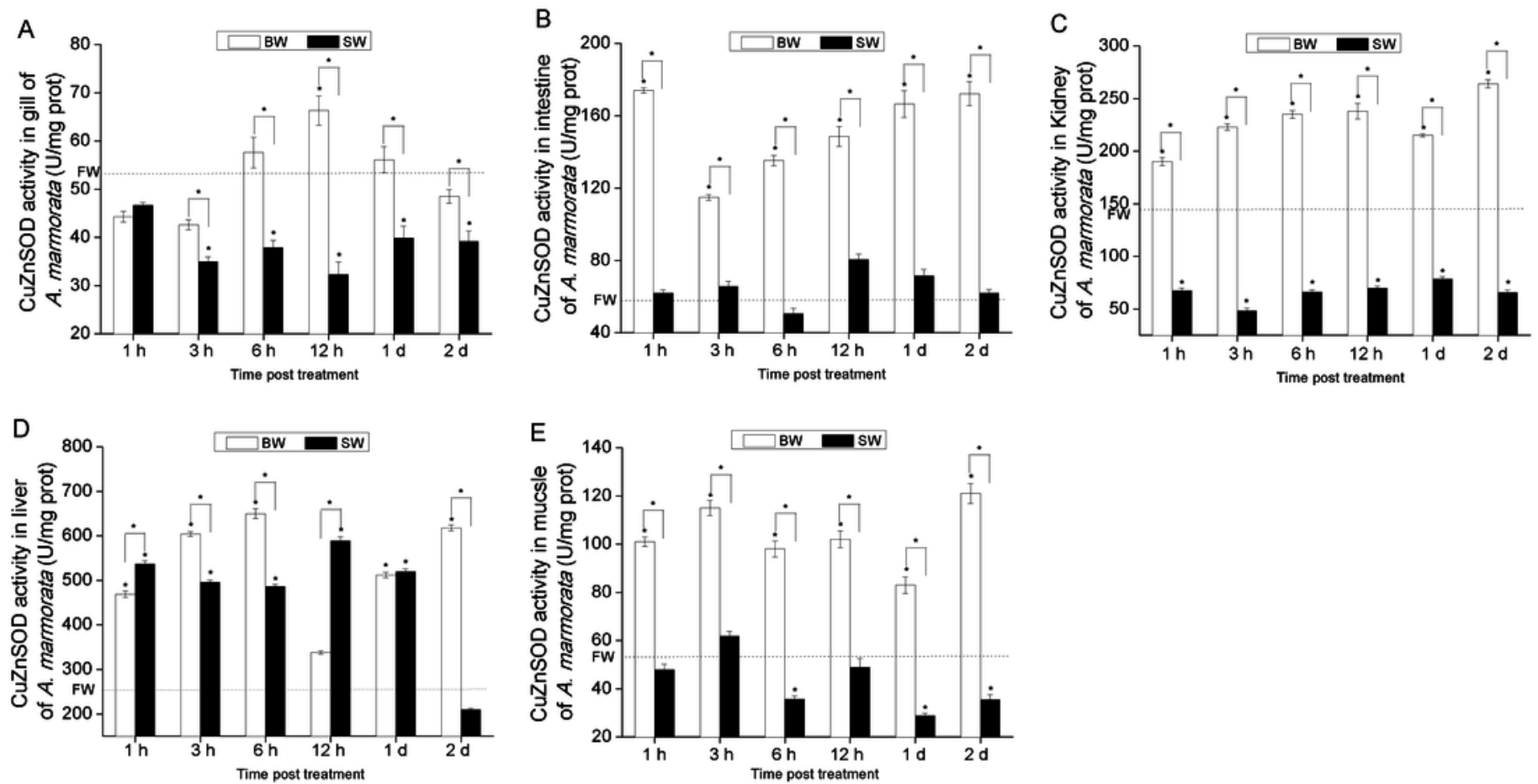